\title{
Utilidad de un modelo informatizado para estratificación de riesgo y decisión de lugar de tratamiento en la neumonía de la comunidad
}

\author{
E. TABERNERO HUGUET, J.L. LOBO BERISTAIN, M. SÁNCHEZ FERNÁNDEZ1, \\ P. LOPETEGUI ERASO ${ }^{1}$, V. ZORRILLA, P. P. ESPAÑA YANDIOLA ${ }^{2}$
}

Servicios de Neumología y ${ }^{1}$ Urgencias. Hospital Txagorritxu.Vitoria-Gasteiz. ${ }^{2}$ Servicio de Neumología. Hospital de Galdakao. Vizcaya

EVALUATION OF A COMPUTER PREDICTIVE MODEL TO STRATIFY RISK AND SITE OF CARE IN COMMUNITY ACQUIRED PNEUMONIA

\section{RESUMEN}

Introducción: La neumonía adquirida en la comunidad (NAC) es una patología muy común y con una gran variabilidad en la práctica clínica. La decisión de hospitalizar o no a un paciente es la más importante en cuanto al nivel de tratamiento y costes. Recientemente un hospital de nuestra comunidad (Hospital de Galdakao) ha desarrollado una regla predictiva basada en el Pneumonia Severity Index (PSI), modificado por varios criterios adicionales (hipoxemia $<60$, shock, fracaso de tratamiento previo correcto, problemas sociales o incapacidad para la ingesta oral, derrame pleural o comorbilidad inestable) mediante un sencillo programa informático para ayudar en la decisión de ingresar o no a un paciente.

Objetivos: Evaluar la utilidad de ese programa informático en el servicio de urgencias de nuestro hospital.

Resultados: Incluimos de forma prospectiva 662 pacientes con NAC que acudieron a urgencias del Hospital de Txagorritxu, entre Diciembre 2002 y Diciembre 2004. De ellos 58 tuvieron un diagnóstico final diferente. El $47 \%$ (285) fueron tratados de forma ambulatoria. La tasa de reingresos en este grupo fue del $6 \%$ y no hubo ningún éxitus. Fueron hospitalizados 319 pacientes, de ellos 97 pertenecían a clases de riesgo bajo del PSI, 61 de los cuales ingresaron por los criterios adicionales elegidos por nosotros, sufriendo el $45 \%$ de ellos complicaciones significativas. Estos resultados son similares a los obtenidos en el estudio del hospital de Galdakao.

Conclusiones: La aplicación de este programa informático para estratificar riesgo y ayudar a la decisión de ingreso en la NAC es simple, útil, seguro y puede ser exportado a diferentes ámbitos clínicos. Son necesarios criterios adicionales al PSI para detectar los pacientes de bajo riesgo que tienen alguna complicación.

PALABRAS CLAVE: Neumonía adquirida en la comunidad. Hospitalización. Urgencias. Regla predictora de riesgo.

\section{ABSTRACT}

Background: CAP is a common disorder with a great variability in clinical practice. The decision regarding the appropriate site of care is the most important for the level of treatment and costs. Recently a hospital in our region (Hospital de Galdakao) developed a prediction rule based on the Pneumonia Severity Index (PSI) plus some additional criteria (hypoxemia $<60$, shock, previous correct treatment failure, social problems or inability to maintain oral intake, pleural effusion or unstable comorbidities) with an easy computer program to classify patients to be hospitalized or not.

Objective: Evaluate that computer program in the emergency department of our hospital.

Results: We included between December 02 and December 04662 prospective patients with CAP admitted to our emergency department, 58 had a different final diagnosis. 285 (47\%) were treated on outpatient basis. Readmission rate was 6\%. There was no mortality in this group. 319 (53\%) patients were hospitalized, 97 were PSI low risk patients (III), 61 of them were admitted to hospital because additional criteria. $45 \%$ of these "low risk patients" had significant complications. These results are similar to those obtained in Galdakao*

Conclusions: The application of this computer risk stratifying program to assess admission to hospital in CAP is simple useful, secure and can be export to different settings. Additional criteria to PSI are necessary to detect low risk patients that complicate.

KEY WORDS: Clinical prediction rule. Hospitalization. Community acquired pneumonia. Emergency department.

Tabernero Huguet E, Lobo Beristain JL, Sánchez Fernández M, Lopetegui Eraso P, Zorrilla V, España Yandiola PP. Utilidad de un modelo informatizado para estratificación de riesgo y decisión de lugar de tratamiento en la neumonía de la comunidad. An Med Interna (Madrid) 2007; 24: 120-124.

\section{INTRODUCCIÓN}

La neumonía es un proceso muy frecuente con incidencia descrita entre 3-11/1000 habitantes y año. Pese a tratarse de una enfermedad con evolución bien conocida continua existiendo una gran variabilidad en la práctica clínica.

La decisión más importante a tomar durante el manejo del paciente con neumonía es el lugar donde va a ser tratado, tan-

Trabajo aceptado: 21 de noviembre de 2006 
to por nivel de vigilancia, tratamiento y pruebas complementarias como por los costes que conlleva. El lugar donde se toma esa decisión son los servicios de Urgencias hospitalarios, donde una amplia parte del personal que trabaja son médicos residentes en formación con experiencia clínica previa limitada.

En los últimos años han sido descritas y ampliamente validadas, varias reglas predictoras de riesgo de mortalidad en la neumonía de la comunidad, basándose en datos de la historia clínica, exploración física, y pruebas complementarias relativamente sencillas, pero cuyo uso aún no está muy extendido en la práctica clínica habitual. Asimismo el uso de programas informáticos para ayudar en las decisiones de ingreso y tratamiento en neumonía ha sido referido en los últimos años por varios autores $(1,3,6)$

En el 2003 el hospital de Galdakao de nuestra comunidad publicó los resultados de la aplicación de un modelo informático basado en la regla de riesgo de Fine o PSI, uno de los índices pronósticos más aceptados universalmente, que consta de 20 ítems a los cuales se asignan unos puntos determinados, basados en la edad, antecedentes, datos de la exploración física, analítica y radiología, clasificando al paciente en cinco clases de riesgo o gravedad según la puntuación final obtenida. Este modelo además, incluye una serie de factores modificadores, para ayudar en la decisión de ingresar al paciente o no $(1,2)$.

El objetivo del presente trabajo fue evaluar la utilidad de ese programa informático en nuestro medio, concretamente en el Servicio de Urgencias del Hospital de Txagorritxu (Vitoria, Alava), un hospital general de 480 camas.

\section{MATERIAL Y MÉTODOS}

Definimos neumonía como la aparición de un nuevo infiltrado en la radiografía, (valorada por un radiólogo) junto con la presencia de síntomas de infección respiratoria. Se excluyeron pacientes con inmunodepresión (corticoides $>10$ $\mathrm{mg} /$ día más de un mes, trasplantados, quimioterapia en los seis meses previos e infección VIH) y menores de 14 años. Los pacientes ingresados en los 15 días previos también fueron excluidos.

El programa informático de estratificación de riesgo incluía la escala de Fine, y aconsejaba ingresar al paciente con puntuación mayor de 90, hospitalización a domicilio entre 7090 si era posible por lugar de residencia y disponibilidad de horario o ingreso en caso contrario. Los pacientes con puntuación inferior a 70 y sin factor de riesgo eran dados de alta, recibiendo una llamada telefónica para valorar evolución a las 72 horas y si no presentaban complicaciones se citaban automáticamente para control en un mes en consulta externa del Servicio de Respiratorio.

Realizamos una pequeña modificación a la regla de FINE y en aquellos pacientes con $\mathrm{SaO}_{2}>92 \%$ respirando aire ambiente no se les realizó gasometría arterial.

Se añadió una serie de criterios modificadores, cuya presencia era considerada indicativa de ingreso independientemente de la puntuación obtenida en la escala de Fine. Estos factores fueron: derrame pleural significativo, insuficiencia respiratoria $\left(\mathrm{pO}_{2}<60\right)$, inestabilidad hemodinámica, comorbilidad descompensada, problema social o incapacidad para ingesta oral y falta de respuesta a tratamiento anti- biótico correcto mantenido 48-72 horas. El programa también sugería tratamiento antibiótico indicado de acuerdo a las normativas SEQ-SEPAR que estaban vigentes en ese momento (4).

El tiempo para completar los ítems de cada caso en el ordenador supuso una media de tres minutos.

En el seguimiento se recogieron, de forma prospectiva, datos del ingreso, cambio de antibiótico, visitas al servicio de urgencias o consulta precoz en neumología, mortalidad o reingreso en los 30 días siguientes, curación radiológica y complicaciones. Se consideró complicación la presencia de una de las siguientes situaciones: intolerancia a medicación, descompensación de comorbilidad, shock, derrame pleural significativo que precisó toracocentesis o drenaje, fracaso renal, sobreinfección e insuficiencia respiratoria mantenida más de 24h, y otra menos frecuentes como hemorragia digestiva, TEP, CID, situaciones todas que justifican prolongación de estancia hospitalaria provocando cambios en tratamiento $\mathrm{u}$ otras actuaciones medicas.

Se realizó estudio estadístico, con análisis descriptivo de las principales características de la muestra utilizando medias y desviación estandar (DE) en las variables continuas y porcentajes en las categóricas. Para la comparación de distintas características entre dos grupos se utilizó la prueba de la Chi cuadrado o el test exacto de Fisher para variables cualitativas y la t de Student o la U de Mann-Whitney para las variables cuantitativas. Se consideró significativo un valor de $\mathrm{p}<0,05$.

\section{RESULTADOS}

Se incluyeron 662 pacientes consecutivos entre el 15 de diciembre del 2002 y 15 de diciembre del 2004 diagnosticados de neumonía en el Servicio de Urgencias. De ellos 58 tuvieron que ser excluidos en un análisis posterior por tener un diagnóstico final distinto (bronquiectasias 18, insuficiencia cardiaca 10, infección respiratoria en EPOC 8, neoplasia 6, y otras como TEP o bronquiolitis obliterante en menor número).

Por lo tanto se analizaron 604 pacientes cuyas características demográficas se muestran en la tabla I.

Se trataron de forma ambulatoria 285 pacientes (47\%), 33 de ellos mediante hospitalización a domicilio, los 319 restantes fueron ingresados.

\begin{tabular}{|c|c|c|}
\hline \multicolumn{3}{|c|}{ TABLA I } \\
\hline \multicolumn{3}{|c|}{$\begin{array}{c}\text { CARACTERÍSTICAS DEMOGRÁFICAS DE LOS PACIENTES } \\
\text { CON NAC (INGRESADOS Y NO INGRESADOS) }\end{array}$} \\
\hline & $\begin{array}{l}\text { Nuestra cohorte } \\
\quad n=604\end{array}$ & $\begin{array}{l}\text { Cohorte de Galdakao } \\
\qquad n=616\end{array}$ \\
\hline Edad media (DE) & $57(21,5)$ & \\
\hline Pacientes < 50 años & $224(37 \%)$ & $30,4 \%$ \\
\hline Sexo & 247 mujeres (40\%) & $36 \%$ \\
\hline Clase riesgo PSI I & $197(32 \%)$ & $29 \%$ \\
\hline PSI II & $146(24 \%)$ & $16,6 \%$ \\
\hline PSI III & $115(19 \%)$ & $18,7 \%$ \\
\hline PSI IV & $110(18 \%)$ & $23 \%$ \\
\hline PSI V & $36(6 \%)$ & $12 \%$ \\
\hline
\end{tabular}


De los pacientes tratados de forma ambulatoria, la tasa de reingreso fue del 6\% (18 pacientes), 46 acudieron de nuevo a Urgencias o precisaron una revisión precoz, de los cuales 35 (12\%) necesitaron un cambio de antibiótico, pero al no existir deterioro clínico radiológico no fueron ingresados. En la tabla II se analizan los antibióticos usados, si fueron necesarios cambios y el motivo de estos (fracaso de tratamiento o intolerancia). No hubo ningún éxitus en este grupo.

TABLA II

RESULTADOS DE TRATAMIENTO ANTIBIÓTICO EN PACIENTES AMBULATORIOS

\begin{tabular}{lcccc}
\hline Tto. antibiótico & Total & Intolerancia & $\begin{array}{c}\text { Fracaso } \\
\text { tratamiento }\end{array}$ & Reingreso \\
\hline $\begin{array}{l}\text { Amoxicilina 0 } \\
\text { amoxiclavulanico }\end{array}$ & 95 & 3 & 6 & 5 \\
\hline Claritomicina & 54 & 3 & 6 & 7 \\
\hline $\begin{array}{l}\text { Levofloxacino 0 } \\
\text { moxifloxacino }\end{array}$ & 120 & 8 & 7 & 5 \\
\hline Otros & 16 & 0 & 1 & 1 \\
\hline Total & 285 & 14 & 20 & 18 \\
\hline
\end{tabular}

Ingresaron 319 pacientes, 7 precisaron UCI (2\%). La estancia media fue de 8,06 $\pm 5,9$ días. La tasa de complicaciones según las hemos definido fue del 56\%, 179 pacientes. Reingresaron 32 pacientes en los 30 días siguientes al alta, un 10\%. La mortalidad global a los 30 días fue del 8\% (26 pacientes). En las tablas III y IV se muestran estos datos detallados según el antibiótico utilizado y la clase de riesgo de Fine.

Se realizó estudio estadístico analizando los datos a la luz del antibiótico utilizado sin observar ninguna diferencia respecto a mortalidad, necesidad de cambio de tratamiento, reingresos o complicaciones.

De los pacientes que ingresaron, 97, (30\% de todos los ingresados) pertenecían a las clases I y II de bajo riesgo de Fine, de los cuales 61 presentaban alguno de los criterios modificadores de ingreso predefinidos por nosotros, en el resto no se pudo hallar a priori una causa clara de ingreso.

TABLA III

RESULTADOS DE TRATAMIENTO ANTIBIÓTICO EN INGRESADOS GLOBAL

\begin{tabular}{lcccc}
\hline Tto. antibiótico & Total & Cambio tto. & Mortalidad & Complicaciones \\
\hline Amoxiclavulánico & 77 & 11 & 5 & 40 \\
\hline Ceftriaxona & 34 & 5 & 6 & 22 \\
\hline $\begin{array}{l}\text { Cefriaxona 0 am-c+ } \\
\text { claritomicina }\end{array}$ & 64 & 8 & 7 & 39 \\
\hline Levofloxacino & 124 & 16 & 6 & 66 \\
\hline Otros & 20 & 5 & 2 & 12 \\
\hline
\end{tabular}

\section{TABLA IV}

RESULTADOS DE PACIENTES INGRESADOS POR GRUPO DE RIESGO

\begin{tabular}{lccccc}
\hline & I-II & $I I I$ & IV & V & Total \\
\hline $\mathrm{N}=$ & 97 & 81 & 106 & 35 & 319 \\
\hline Estancia media (+/- DE) & $6,6 \pm 4,4$ & $8,6 \pm 6,4$ & $8,8 \pm 6,5$ & $9,05 \pm 5,8$ & $8,06 \pm 5,9$ \\
\hline Reingreso & 5 & 10 & 11 & 6 & 32 \\
\hline Cambio en tratamiento & 12 & 10 & 15 & 8 & 45 \\
\hline Complicaciones & 33 & 42 & 74 & 30 & 179 \\
\hline UCl & 3 & 1 & 1 & 2 & 7 \\
\hline Exitus & 0 & 2 & 13 & 11 & 26 \\
\hline
\end{tabular}

Si analizamos por separado estos dos grupos de pacientes de bajo riesgo ingresados, se observa que el grupo que ingresó por la presencia de alguno de los criterios predefinidos presentó una tasa de complicaciones estadísticamente significativa mayor (test de Fisher) que los que no presentaron estos criterios. La necesidad de UCI y estancia media también fue mayor aunque sin diferencias estadísticas (Tabla V).

\section{TABLA V}

PACIENTES DE BAJO RIESGO INGRESADOS. DIFERENCIAS SEGÚN EL INGRESO POR CRITERIOS MODIFICADORES O NO

\begin{tabular}{lccc}
\hline & Criterios presentes & Criterios ausentes & $p$ \\
\hline Numero total $=97$ & 61 & 36 & \\
\hline Estancia media & 6,8 & 6 & 0,35 \\
\hline Mortalidad & 0 & 0 & - \\
\hline Ingreso en UCl & $3 / 4,9 \%$ & 0 & 0,5 \\
\hline Reingresos & $2 / 3,2 \%$ & $3 / 8,6 \%$ & 0,6 \\
\hline Complicaciones & $26 / 42 \%$ & $7 / 19 \%$ & $0,009^{*}$ \\
\hline
\end{tabular}

*Estadísticamente significativo.

\section{DISCUSIÓN}

El interés de este trabajo es evaluar en nuestro medio la utilidad de una herramienta informática en el manejo de una patología bien conocida en el servicio de urgencias como es la neumonía, donde parte de los médicos que toman las decisiones mas importantes inicialmente son residentes, con limitada experiencia clinica (hasta un $64 \%$ en el trabajo de Menéndez y cols.) (5), un colectivo que además suele mostrar adherencia al empleo de guías clínicas ( $82 \%$ en el mismo trabajo).

De nuestros datos llama la atención, en primer lugar, el alto número de diagnósticos finales distintos de neumonía $(8,7 \%)$ pese a la valoración por un radiólogo, aunque inferior al $11 \%$ de pacientes ingresados con diagnostico de neumonía que posteriormente no se confirma referido en otros estudios (6).

En los pacientes con neumonía la distribución por clase de Fine es muy similar a otros trabajos, quizá con un porcentaje más bajo de pacientes en el grupo $\mathrm{V}$, los más graves $(1,3,6,7,22)$ lo que nos permite hasta cierto punto comparar datos (Tabla I). 
Del total de pacientes que acude a urgencias, un $47 \%$ es tratado de forma ambulatoria, de ellos reingresan 17 , un $6 \%$. Un $11 \%$ necesitó además cambios en el tratamiento, lo que nos recuerda la importancia de un seguimiento estrecho inicial en las primeras 48-72 horas o de dar unas instrucciones claras al paciente sobre cuando debe realizar una nueva consulta médica. En el trabajo original de España, Capelastegui y cols. del Hospital de Galdakao (1) el 35\% de los pacientes fueron tratados de forma ambulatoria. Recientemente este mismo grupo ha publicado una disminución del porcentaje de ingreso de los pacientes que acuden a Urgencias a lo largo del tiempo hasta un 56,5\% tras cuatro años de la implantación de una guía clínica (14).

Respecto a los pacientes ingresados la tasa de mortalidad global es similar a la descrita en la literatura, entre el 5 y $13 \%$ $(1,5-8,13,24)$. El porcentaje de fracasos de tratamiento es también comparable a la de otros trabajos realizados en nuestro medio, $15 \%$ en el trabajo de Menéndez (20). La estancia media es claramente superior a la del grupo de Galdakao, aunque similar a otros resultados de un estudio multicéntrico en nuestro país, 9 días (13).

Sin embargo, la tasa de complicaciones, a pesar de que hemos incluido sólo situaciones ya descritas en otros trabajos que retrasan el alcanzar la estabilidad clínica justifican y prolongan la estancia hospitalaria, es claramente superior a lo descrito por otros autores, incluido el grupo de Galdakao, cuyas tasas oscilan entre el 21 y $29 \%(22,23,29)$ desconocemos la razón, quizá por una estricta selección de pacientes ingresados o por una diferente definición.

Si analizamos los pacientes por clases de riesgo de Fine, ingresa un $30 \%$ de pacientes de clases de riesgo bajo (I y II) frente a un $25 \%$ en el trabajo Galdakao y hasta un $57 \%$ en otros estudios publicados $(12,15,16,21,23)$. Otros autores ya describen que pese a que la aplicación de una guía clínica permite reducir el porcentaje de pacientes de bajo riesgo ingresados, sigue existiendo un cierto número de ellos que precisa ingreso y que incluso se complica, justificando que las guías clínicas sean sólo una orientación y no un sustituto del juicio clínico. De hecho, en un estudio de la BTS un $27 \%$ de los pacientes que ingresan en UCI por neumonía pertenecen a clases de riesgo bajo (17). Dos estudios controlados encontraron que, al aplicar una guía clínica ingresa un porcentaje menor de pacientes de clase I-III, persistiendo todavía un $42-31 \%$ de pacientes de estos grupos que ingresa $(15,16)$. Además en otro trabajo de los mismos autores, el 19\% de los pacientes de bajo riesgo que acuden a los servicios de Urgencias de 6 hospitales ingresa, de ellos el $48 \%$ permanece $>5$ días y el 19\% sufre una o mas complicaciones, algunas graves como fracaso ventilatorio y empiema, concluyendo que hacen falta mejores criterios para guiar el ingreso de un paciente.

Lo mismo ocurre en un estudio realizado en nuestro país, donde el $40 \%$ de los pacientes ingresados pertenecían a clases de riesgo bajas y de ellos, el $60 \%$ necesitó oxígeno o tuvo complicaciones pleurales (29).

En nuestros datos, reevaluando la historia clínica de los pacientes de bajo riesgo que ingresan, no podemos encontrar clara causa de ingreso (salvo presión del paciente o familiares) en un $28 \%$ (16-36\% en otros estudios) (14,21). El resto ingresa por los criterios modificadores indicativos de ingreso añadidos previamente, criterios que son imperativo de tratamiento hospitalario en algunos algoritmos $(4,21,27)$, causa de exclusión de tratamiento ambulatorio u hospitalización a domicilio en otros trabajos (15,27-28), o criterios de severidad en alguna normativa, como la de la BTS del 2001 (30) y también relacionados con prolongación de la estancia (13). En algunos estudios hasta un tercio de los pacientes de los grupos I-III presentan estos criterios indicativos de ingreso (26), y suponen hasta un $27 \%$ en otro trabajo del grupo de Galdakao (11).

Quisimos investigar si había diferencias entre el grupo de pacientes ingresados por estos criterios modificadores y los que lo hicieron sin aparente razón. No pudimos encontrar diferencias en mortalidad y reingresos, pero sí encontramos diferencias significativas en el número de complicaciones o eventos que justifican estancia e intervención médica, así como una tendencia clara superior en la necesidad de ingreso en UCI, probablemente no significativa por el escaso número de pacientes que va a UCI en este trabajo. Esto nos lleva a sugerir que la presencia de uno de estos criterios pudiera ser por si misma indicación de ingreso, como así aparece implícitamente en algunas guías apoyando la idea de que el riesgo de muerte (que es lo que mide la regla predictora de Fine) puede no ser igual a la necesidad de hospitalización.

En conclusión, la similitud de los grupos de riesgo en las cohortes nos permite comparar los resultados de la aplicación de este programa informático de estratificación de riesgo en nuestro medio con los resultados del Hospital de Galdakao observando que son muy similares tanto en mortalidad como en porcentaje de pacientes tratados de forma ambulatoria y reingresos. La utilización de esta aplicación informática es útil, sencilla segura y puede ser exportada a diferentes ámbitos. Parecen necesarios criterios adicionales al PSI para detectar pacientes de bajo riesgo que se complican.

\section{Bibliografía}

1. España PP, Capelastegui A, Quintana JM, Soto A, Gorordo I, GarciaUrbaneja M, et al. A prediction rule to identify allocation of inpatient care in community-acquired pneumonia. Eur Respir J 2003; 21: 695701.

2. Fine MJ, Auble TE, Yealy DM, Hanusa BH, Weissfeld LA, Singer $\mathrm{DE}$, et al. A prediction rule to identify low-risk patients with community acquired pneumonia. N Engl J Med 1997; 336: 243-50.

3. Wright AA, Maydom BW. Improving the implementation of community acquired pneumonia guidelines. Intern Med J 2004; 34: 507-9.

4. Normativa de la Sociedad Española de Quimioterapia- Sociedad Española Patología del Aparato Respiratorio (SEQ-SEPAR). Frias J,
Gomis M, Prieto J, Mensa J, Bouza M, Garcia Rodríguez JA, et al. Initial empirical antibiotic treatment of community acquired pneumonia. Rev Esp Quimioter 1998; 11: 255-61

5. Menéndez R, Torres A, Zalacain R, Aspa J, Martín Villasclaras JJ, Borderías L, et al. Guidelines for the treatmentof community acquired pneumonia: predictors of adherence. Am J Respir Crit Care Med 2005; 172: 757-762.

6. Buising KL, Thursky KA, Black JF, Mc Gregor L, Street AC, Kennedy MP. A prospective comparison of severity scores for identifying patients with severe community acquired pneumonia: reconsidering what is meant by severe pneumonia. Thorax 2006; 61: 419-424. 
7. Marrie TJ, Lieling Wu. Factors influencing in-hospital mortality in community acquired pneumonia. Chest 2005; 127: 1260-1270.

8. Kaplan V, Angus DC, Griffin MF, Clermont G, Scott Watson R, et al. Hospitalized community acquired pneumonia in the elderly: age and sex related patterns of care and outcome in the United States. Am J Respir Crit Care Med 2002; 165: 766-772.

9. Meeham TP, Fine MJ, Krumhol HM, Scinto JD, Galusha DH, Mockalis JT, et al. Quality of care process and outcomes in elderly patients with pneumonia. JAMA 1997; 278: 2080-2084.

10. Capelastegui A, España PP, Quintana JM, Gorordo I, Gallardo MS, Idioaga I, et al. Management of community acquired pneumonia and secular trends at different hospitals. Respir Med 2005; 99: 268-78.

11. Capelastegui A, España PP, Quintana JM , Areitio I, Gorordo I, Egurrola M, et al. Validation of a predictive rule for the management of community acquired pneumonia Eur Respir J 2006 ; 27: 151-157.

12. Aliyu ZH, Aliyu MH, Mc Cormick K. Determinants for hospitalization in "low risk" community acquired pneumonia. BMC Infect Dis 2003; 3: 11

13. Menéndez R, Cremades MJ, Martínez-Moragón E, Soler JJ, Reyes S, Perpiña M, et al. Duration of stay in pneumonia: influence of clinical factors and hospital type. Eur Respir J 2003; 22: 643-648.

14. Mandell L. Decisions about treating community acquired pneumonia. Ann Intern Med 2005; 142: 215-16

15. Atlas SJ, Benzer TI, Borowsky LH Chang Y, Burnham Dc, Metlay JP, et al. Safetly increasing the proportion of patients with community acquired pneumonia treated as outpatients: an interventional trial. Arch Intern Med 1998; 158: 1350-6.

16. Marrie TJ, Lau CY, Wheeler SL, Wong CJ, Vandervoort MK, Feagan BG: for the CAPITAL Study Investigatiors. A controlled trial of a critical pathway for treatment of community acquired pneumonia. JAMA 2000; 283: 749-55.

17. Angus DC, Marrie TJ, Obrosky D, Clermont G, Dremsizov TT, Coley $\mathrm{C}$, et al. Severe community acquired pneumonia: use of intensive care services and evaluation of American and British Thoracic Society Diagnostic criteria. Am J Respir Crit Care Med 2002; 166: 717-23.

18. Van der Eerden MM, de Graaff S, Bronsveld W, Jansen HM, Boersma WG. Prospective evaluation of pneumonia severity index in hospitalized patients with community acquired pneumonia. Respir Med 2004; 98: 872-8.
19. Ridley PD, Aronsky D, Dean NC. Validation of the 2001 ATS criteria for severe community acquired pneumonia . Crit Care Med 2004; 32,12: 2398-402.

20. Menéndez R, Torres A, Zalacain R, Aspa J, Martin Villasclaras JJ, Borderias L, et al. Risk factors of treatment failure in community acquired pneumonia: implications for disease outcome. Thorax 2004; 59: 960-965.

21. Arnold FW, Ramirez JA, McDonald LC, Xia EL. Hospitalization for community acquired pneumonia. The Pneumonia Severity Index vs. Clinical Judgement. Chest 2003; 124: 121-4.

22. Menéndez R, Torres A, Rodríguez de Castro F, Zalacain R, Aspa J, Martin Villasclaras JJ, et al. Reaching stability in community acquired pneumonia; The effects of the severity of disease, treatment, and the characteristics of patients. Clin Infect Dis 2004; 39: 1783-90.

23. Roson B, Carratalá J, Dorca J, Casanova A, Manresa F, Gudiol F. Etiology, reasons for hospitalization, risk classes and outcomes of community acquired pneumonia in patients hospitalized on the basis of conventional admission criteria. Clin Infect Dis 2001; 33: 158-65.

24. Vecchiarino P, Bohannon RW, Ferullo J, Maljanian R. Short-Term outcomes and their predictors for patients hospitalized with community acquired pneumonia . Heart Lung 2004; 33: 301-7.

25. Ewig S, de Roux A, Bauer T, García E, Mensa J, Niederman M, Torres A. Validation of predictive rules and indices of severity for community acquired pneumonia. Thorax 2004; 59: 421-427.

26. Marrie TJ, HuangJQ. Low risk patients admitted with community acquired pneumonia. Am J Med 2005; 118: 1357-63.

27. Carratalá J, Fernandez-Sabe N, Ortega L, Castellsague X, Rosón B, Dorca J, et al. Outpatient care compared with hospitalization for community acquired pneumonia. Ann Intern Med 2005; 142: 165-172.

28. Metlay JP, Fine MJ. Testing strategies in the initial management of patients with community acquired pneumonia. Ann Intern Med 2003; 138: 109-118.

29. Roson B, Carratalá J, Fernández-Sabe N, Tubau F, Manresa F, Gudiol F. Causes and factors associated with early failure in hospitalized patients with community acquired pneumonia. Arch Intern Med 2004; 164: 502-508.

30. British Thoracic Society Standards of Care Committe. BTS guidelines for the management of community acquired pneumonia in adults. Tho$\operatorname{rax} 2001 ; 56$ (Supl. IV): iv1-64. 